TRANSACTIONS OF THE

AMERICAN MATHEM ATICAL SOCIETY

Volume 186, December 1973

\title{
CENTRAL IDEMPOTENT MEASURES ON COMPACT GROUPS
}

BY

\section{DANIEL RIDER( 1 )}

\begin{abstract}
Let $G$ be a compact group with dual object $\Gamma=\Gamma(G)$ and let $M(G)$ be the convolution algebra of regular finite Borel measures on $G$. The author has characterized the central idempotent measures on certain $G$, including the unitary groups, in terms of the hypercoset structure of $r$. The characterization also says that, on certain $G$, a central idempotent measure is a sum of such measures each of which is absolutely continuous with respect to the Haar measure of a closed normal subgroup. The main result of this paper is an extension of this characterization to products of certain groups. The known structure of connected groups and a recent result of Ragozin on connected simple Lie groups will then show that the characterization is valid for connected groups. On the other hand, a simple example will show it is false in general for nonconnected groups. This characterization was done by Cohen for abelian groups and the proof borrows extensively from Amemiya and Itô's simplified proof of Cohen's result.
\end{abstract}

1. Canonical measures. Throughout the paper $G$ will be a compact group. The dual object $\Gamma$ of $G$ is the set of equivalence classes of irreducible unitary representations of $G$. For $a \in \Gamma, \chi_{a}$ will denote the character of the class and $d(a)$ its degree. For ease of notation we define $\Psi_{a}=\chi_{a} / d(a)$. A measure $\mu \epsilon$ $M^{Z}(G)$, the center of $M(G)$, has a Fourier-Stieltjes transform

$$
\hat{\mu}(a)=\int \bar{\Psi}_{a} d \mu \quad(a \in \Gamma) .
$$

$\mu$ is idempotent, that is $\mu * \mu=\mu$, provided $\hat{\mu}(\alpha)$ is always 0 or $1 . J(G)$ will denote the class of central idempotent measures on $G$.

If $H$ is a closed subgroup of $G$ let $\mathbb{M}_{H}$ denote the normalized Haar measure of $H . \pi_{H}$ is idempotent; $\mathbb{M}_{H} \in J(G)$ provided $H$ is normal.

It is convenient to consider a larger class

$$
F(G)=\left\{\mu \in M^{Z}(G): \hat{\mu}(\alpha) \text { is an integer }\right\} .
$$

Received by the editors March 12, 1973.

AMS (MOS) subject classifications (1970). Primary 22C05, $43 A 05$; Secondary 43440.

Key words and phrases. Central measure, idempotent measure, hypergroup, hypercoset ring.

(1) This research was supported in part by NSF Grant GP-24182. The author is a fellow of the Alfred P. Sloan Foundation.

Copyright $\odot$ 1974, American Mathematical Society 
$F(G)$ consists then of those central measures $\mu$ that satisfy $P(\mu)=0$ for some polynomial $P$ with integral roots (for a noncompact $G, F(G)$ will be defined in this way). For $\mu \in F(G)$ we let $E(\mu)=\{a: \hat{\mu}(\alpha) \neq 0\}$.

The hypercoset structure of $\Gamma$ is described in [5]. If $H$ is a closed normal subgroup of $G$ then $E\left(\pi_{H}\right)=\left\{a:\left.\Psi_{a}\right|_{H} \equiv 1\right\}=H^{\perp}$ is a normal subhypergroup of $\Gamma$. If $\beta \in \Gamma$ the hypercoset $\beta H^{\perp}$ consists of the $a \in \Gamma$ such that $\chi_{a}$ appears in the decomposition of $\chi_{\beta} \chi_{\gamma}$ for some $\gamma \in H^{\perp}$. Also $\beta H^{\perp}=\left\{\alpha:\left.\Psi_{\alpha}\right|_{H}=\left.\Psi_{\beta}\right|_{H}\right\}$. The hypercoset ring of $\Gamma$ is the smallest ring of sets containing all hypercosets.

There are two ways to attempt to characterize the measures in $F(G)$.. First, for $\mu \in F(G)$ and $n$ an integer let $E_{n}(\mu)=\{\alpha: \hat{\mu}(\alpha)=n\}$. If $\mu \in J(G)$ then $E_{1}(\mu)$ $=E(\mu)$. It is show $\mathrm{n}$ in [5] that every set in the hypercoset ring of $\Gamma$ is $E(\mu)$ for some $\mu \in J(G)$ and that for certain groups the converse is also true. This implies, for such groups, that for $\mu \in F(G)$ each $E_{n}(\mu)$ is in the hypercoset ring.

Second, some measures in $F(G)$ arise naturally from well-known measures on $G$.

Definition 1.1. A measure $\mu$ is canonical if

$$
\mu=\sum_{a} n_{\alpha} c_{\alpha} d(\alpha) \chi_{\alpha} \pi_{H_{a}}
$$

where the sum is finite, $n_{a}$ is an integer, $H_{a}$ is a closed normal subgroup and

$$
\frac{1}{c_{a}}=\int\left|\chi_{a}\right|^{2} d \pi_{H_{a}}
$$

The following lemma, which connects the two concepts above, is an immediate consequence of Theorem 1 of $[1]$ and the fact that the intersection of two hypercosets is a finite union of hypercosets.

Lemma 1.2. (a) Every canonical measure is in $F(G)$.

(b) A measure $\mu \in F(G)$ is canonical if and only if eacb $E_{n}(\mu)$ belongs to the bypercoset ring of $\Gamma$.

We will use the usual notation $\mu<<\nu$ to indicate that $\mu$ is absolutely continuous with respect to $\nu$. It is easy to see

Lemma 1.3. $\mu \in F(G)$ is canonical if and only if there are finitely many closed normal subgroups $H_{i}$ and $\mu=\Sigma_{\mu_{i}}$ with $\mu_{i}<<M_{H_{i}}$

2. The main result. Let $\Gamma_{1}$ denote those $\alpha \in \Gamma$ with $d(\alpha)=1 . \Gamma_{1}$ consists of the complex homomorphisms of $G$ and is the dual group of the abelian group $G / G^{\prime}$ where $G^{\prime}$ is the commutator subgroup of $G$. For $a \in \Gamma_{1}$ we can identify $\alpha$ and $\chi_{a}$. Now if $a, \beta \in \Gamma$ it may happen that the tensor product $\alpha \otimes \beta$ is irreducible. If it is we let $\alpha \beta$ denote $\alpha \otimes \beta$ so that $\chi_{a} \chi_{\beta}=\chi_{\alpha \beta}$. If $a \in \Gamma_{1}$ 
this is always the case. We also let $Z=Z(G)$ denote the center of $G$.

Definition 21. $G$ is said to satisfy condition I provided

$$
\lim _{d(a) \rightarrow \infty} \Psi_{a}(x)=0
$$

for all $x \notin Z$.

$G$ is said to satisfy condition II provided that for each positive integer $t$ there are finitely many irreducible representations $\beta_{1}, \ldots, \beta_{s}$ of degree $t$ such that if $d(\beta)=t$ then $\beta=\alpha \beta_{i}$ for some $i$ and some $\alpha \in \Gamma_{1}$.

It should be noted that groups having open centers, in particular abelian groups and finite groups, satisfy both conditions. In [5] it is shown that unitary groups do also. In $\$ 9$ it will be shown, using a result of Ragozin [4], that every compact connected simple Lie group satisfies both conditions.

We can now state the main result of the paper.

Theorem 2.2. Let $G_{i}(i \in A)$ be compact groups satisfying conditions I and II and let $G=\Pi_{A} G_{i}$ Then every measure in $F(G)$ is canonical.

Together with Lemma 1.2 this then gives

Corollary 23. If $G$ is as above then $E \subset \Gamma$ is $E(\mu)$ for some $\mu \in J(G)$ if and only if $E$ belongs to the bypercoset ring of $\Gamma$.

It is well known (cf. [3, Theorem 2.1.4]) that an idempotent measure of norm 1 on a locally compact group is of the form $\gamma \pi_{H}$ for some compact subgroup $H$ and some $\gamma \in \Gamma_{1}(H)$. It follows that, for any compact group $G$, the elements of $F(G)$ of norm 1 are canonical.

Definition 24. A measure $\mu \in F(G)$ is irreducible if it cannot be written as the sum of two mutually singular nonzero measures in $F(G)$.

Definition 25. The support group $L(\mu)$ of a measure $\mu \in M(G)$ is the smallest closed subgroup that carries $\mu$.

Clearly if $\mu \in M^{Z}(G)$ then $L(\mu)$ is normal. A rough idea of the proof of Theorem 2.2 is to show that if $\mu \in F(G)$ is irreducible then $\mu<<\mathbb{I}_{L(\mu)^{\bullet}}$

The proof of Theorem 2.2 is in $\$ 8$. $\$ 3$ deals with projections of $M(G)$ onto the measures carried by the cosets of a normal Borel subgroup. $\$ 4$ contains results concerning $F(G)$ for an arbitrary compact group $G$. In $\$ 5$ it is shown that if $\mu$ is canonical and $\|\mu\|>1$ then $\|\mu\|>1+1 / 700$. This generalizes a wellknown result on abelian groups and is perhaps of independent interest. $\$ \$ 6$ and 7 contain results about $F(G)$ for $G$ as in the hypotheses of Theorem 2.2. They are an attempt to use the methods of Amemiya and Itô [1] for abelian groups in this more general setting. In $\$ 9$ a result of Ragozin [4] is used to show that the conclusions of Theorem 2.2 and Corollary 2.3 are valid for connected groups. An example of where Theorem 2.2 fails is given in $\$ 10$. 
3. Projections. Let $H$ be a normal Borel subgroup of $G$. For $\mu \in M(G)$ define

$$
\Pi_{H} \mu(E)=\sum_{x} \mu(E \cap H x)
$$

the sum being over distinct coset representatives of $H$.

Lemma 3.1. (a) $\Pi_{H}$ is a bomomorphism of the algebra $M(G)$ into itself.

(b) $\Pi_{H}$ maps $F(G)$ into itself.

Proof. (a) is proved in [7, Theorem 3.4.1] for $H$ a closed subgroup and $G$ abelian. The proof works equally well for this more general situation. Since $H$ is normal it is easily seen that $\Pi_{H}$ maps $M^{Z}(G)$ into itself and (b) then follows since $\Pi_{H}$ is a homomorphism.

The following the orem gives the first indication that an irreducible measure in $F(G)$ is absolutely continuous with respect to the Haar measure of its support group.

Theorem 3.2. Let $\mu \in F(G)$ be irreducible and bave support group L. If $H$ is a closed normal subgroup of $G$ and $\Pi_{H} \mu \neq 0$ then $H \cap L$ is open in $L$.

Proof. Write $\mu=\Pi_{H} \mu+\left(\mu-\Pi_{H} \mu\right)$. By Lemma 3.1 these last two measures are in $F(G)$. Since they are singular and $\mu$ is irreducible we must have $\mu=$ $\Pi_{H} \mu$. Also $\mu=\Pi_{L} \mu$ and it is then easily seen that $\mu=\Pi_{H{ }_{L}} \mu$.

Retopologize $G$ so that the closed subgroup $H \cap L$ is open; let $G$ with this new topology be denoted by $G_{0}$. Since $\mu$ is supported on countably many cosets of $H \cap L$ we have then that $\mu \in F\left(G_{0}\right)$. Now $G_{0}$ is a locally compact group and, since $H \cap L$ is a compact open normal subgroup, $G_{0}$ has small invariant neighborhoods. It follows from [6, Theorem 1] that $\mu$ is supported on a compact subgroup of $G_{0}$. Thus, as an element of $F\left(G_{0}\right), \mu$ is supported on a finite extension $P$ of $H \cap L . P$ is then a closed subgroup of $G$ that carries $\mu$. Hence $L \subset P$ and so $L$ is a finite extension of $H \cap L$; that is $H \cap L$ is open in $L$.

4. $F(G)$ for arbitrary $G$. This section contains some lemmas concerning $F(G)$ for an arbitrary compact group $G$.

Lemma 4.1. If $\mu \in F(G)$ bas support group $L$ and $T=\left\{\left.\Psi_{a}\right|_{L}: a \in E(\mu)\right\}$ is finite then $\mu$ is canonical.

Proof. Let $\left.\Psi_{a_{1}}\right|_{L}, \ldots, \Psi_{\left.a_{t}\right|_{L}}$ be the distinct elements of $T$. Then $E(\mu)=$ $U_{1}^{t} \alpha_{i} L^{\perp}$. These hypercosets are disjoint and, since $L$ carries $\mu, \hat{\mu}$ is constant on each $a_{i} L^{\perp}$. Thus $\mu=\Sigma_{1}^{t} a_{i} \chi a_{i} \prod_{L}$ for some constants $a_{i}$ so that $\mu$ is canonical by Lemma 1.3. 
Lemma 4.2. Let $H$ be a closed normal subgroup of $G$. If $\mu \in F(G)$ and $|\mu|\left(H^{c}\right)<1 / 2$ then $|\mu|\left(H^{c}\right)=0$.

Proof. Let $a$ belong to the hypercoset $\beta \dot{H}^{\perp}$. This implies that $\left.\Psi_{a}\right|_{H}=$ $\left.\Psi_{\beta}\right|_{H}$ so that

$$
|\hat{\mu}(a)-\hat{\mu}(\beta)| \leq \int\left|\bar{\Psi}_{a}-\bar{\Psi}_{\beta}\right| d|\mu| \leq 2|\mu|\left(H^{c}\right)<1
$$

Since $\hat{\mu}$ is integer valued this gives that $\hat{\mu}$ is constant on $\beta H^{\perp}$. It follows easily that $\mu$ is carried by $H$; that is $|\mu|\left(H^{c}\right)=0$.

If $a \in \Gamma$ and $\mu \in F(G)$ then it does not follow that $\Psi_{a} \mu \in F(G)$. The problem of course is that the product of two irreducible characters decomposes, in general, into a sum of several irreducible characters. However a sequence in $\Gamma$ may have the following property.

Definition 4.3. A sequence $\{\alpha\} \subset \Gamma$ is an irreducible sequence if for eacb $\beta \in \Gamma$ there is $\alpha(\beta)$ such that $\alpha \otimes \beta$ is irreducible whenever $a>a(\beta)$.

For example if $G=\Pi_{1}^{\infty} G_{i}$ and $a_{i} \in \Gamma(G)$ is given by some $a_{i} \in \Gamma\left(G_{i}\right)$ then $\left\{a_{i}\right\}$ is an irreducible sequence. This example will be useful in the proof of Theorem 2.2 because if $\{a\}$ is an irreducible sequence and $\mu \in F(G)$ then every weak limit point of $\left\{\Psi_{a}^{\mu}\right\}$ also belongs to $F(G)$.

The following lemma is a generalization of Helson's translation lemma [7, Lemma 3.5.1].

Lemma 4.4. Let $L$ be a closed normal subgroup of $G$. Suppose $\{a\}$ is a sequence in $\Gamma$ with $\Psi_{a} l_{L}$ being distinct. Let $\mu \in M^{Z}(G)$ be carried by $L$. If $\lambda$. is a weak limit point of $\left\{\Psi_{\alpha} \mu\right\}$ then $\lambda$ and $\pi_{L}$ are mutually singular.

Proof. $\left.\chi_{a}\right|_{L}$ decomposes into a sum of irreducible characters on $L$. Since the $\left.\Psi_{a}\right|_{L}$ are distinct any character of $\Gamma(L)$ which appears in $\left.\chi_{a}\right|_{L}$ does not appear in $\left.\chi_{\beta}\right|_{L}$ (for $\alpha \neq \beta$ ). The remainder of the proof follows that of [7, Lemma 3.5.1] exactly.

Lemma 4.5. Let $\mu \in F(G)$ be irreducible. If $\{a\}$ is an irreducible sequence and $\Psi_{a} \mu$ converges weakly to a nonzero canonical measure $\lambda$ then $\mu$ is also canonical.

Proof. Let $L$ be the support group of $\mu . \lambda$ is then also carried by $L$. Now if $H$ is a closed normal subgroup with $\Pi_{H} \lambda \neq 10$ then $\Pi_{H} \mu \neq 10$. By Theorem 3.2 this implies that $H \cap L$ is open in $L$. Thus the Haar measures that appear in the canonical measure $\lambda$ are all absolutely continuous with respect to $\pi_{L}$. By Lemma 4.4 we then have that $\left.\Psi_{a}\right|_{L}$ are the same for $a \geq a_{0}$ so that

$$
\Psi_{a_{0}} \mu=\lambda \text {, }
$$


and

$$
\left|\Psi_{a_{0}}\right|_{L}^{2}=\left.\bar{\Psi}_{a_{0}} \Psi_{a}\right|_{L} \text { for } a \geq a_{0}
$$

We will now show that

$$
\left|\Psi_{a_{0}}\right|_{L} \equiv 1
$$

This gives, by (1), that $\mu<<\lambda$ ' and so $\mu<<\prod_{L}$ which implies, by Lemma 1.3, that $\mu$ is canonical.

Since $\{a\}$ is an irreducible sequence there is $\alpha \geq a_{0}$ so that $\beta=\bar{a}_{0} a$ is irreducible. Because of (2), $\left.\int_{L} \Psi_{\beta} d\right) \prod_{L} \neq 10$ so that $\beta \in L^{\perp}$; that is $\left.\Psi_{\beta}\right|_{L} \equiv 1$. (3) then holds because of (2).

The example of $\$ 10$ will show that we can have $\mu \in F(G)$ being carried by a closed normal subgroup $H$ with $\mu$ canonical when considered as an element of $F(H)$ but not canonical as an element of $F(G)$. However we do have the following.

Lemma 4.6. Let $\mu \in F\left(G_{1} \times G_{2}\right)$ bave support group $L$. Suppose that $L C$ $G_{1} \times K$ where $K$ is a finite normal extension of $Z=Z\left(G_{2}\right)$. Then if $\mu$ is canonical with respect to $G_{1} \times K$ it is also canonical with respect to $G_{1} \times G_{2}$.

Proof. Without loss of generality we can assume that $\mu$ is irreducible as an element of $F\left(G_{1} \times G_{2}\right)$. Now $\mu$ is the sum of finitely many nonzero measures $\mu_{i}$ each of which is absolutely continuous with respect to $\pi_{H_{i}}$, where $H_{i}$ is a closed normal subgroup of $G_{1} \times K$. Now each $H_{i} \subset L$ so that $K_{i}=H_{i} \cap\left(G_{1} \times Z\right)$ is open in $H_{i^{*}}$. Thus $\Pi_{K_{i}} \mu_{i}=\mu_{i^{*}}$. But also $K_{i}$ is normal in $G_{1} \times G_{2}$ and $\Pi_{K_{i}} \mu \neq 1$ 0 so that, by Theorem 3.2, $K_{i}=K_{i} \cap L$ is open in $L$. Thus $H_{i}$ is also open in $L_{i}$ so that $\mu_{i}<<\prod_{H_{i}}<<\prod_{L}$. This implies $\mu<<\prod_{L}$ which, by Lemma 1.3, makes $\mu$ canonical on $G_{1} \times G_{2}$.

The previous lemma can be generalized to infinite products.

Lemma 4.7. Let $G=\Pi_{1}^{\infty} G_{i}$ and let $\mu \in F(G)$ bave support group L. Suppose that $L \subset G_{1} \times \Pi_{2}^{\infty} K_{i}$ where eacb $K_{i}$ is a finite normal extension of $Z_{i}=Z\left(G_{i}\right)$. Then if $\mu$ is canonical with respect to $G_{1} \times \Pi_{2}^{\infty} K_{i}$ it is also canonical witb respect to $G$.

Proof. Without loss of generality we can assume $\mu$ is irreducible as an element of $F(G)$. By Lemma 4.6 we have that, for each $n, \mu$ is canonical with respect to $A_{n}=\Pi_{1}^{n} G_{i} \times \Pi_{n+1}^{\infty} K_{i}$. Thus for each $n$ we can write

$$
\mu=\sum_{j=1}^{a(n)} \mu_{j, n}
$$


where each $\mu_{j, n}$ is absolutely continuous with respect to $\pi_{j, n}$, the Haar measure of $H_{j, n}$, a closed normal subgroup of $A_{n}$. Also $H_{j, n}=L$. We can also assume, for each fixed $n$, that the $\pi_{j, n}$ and hence the $\mu_{j, n}$ are mutually singular. Now $\left\|\mu_{j, n}\right\| \geq 1$ so that $a(n) \leq\|\mu\|$. Thus we can assume that $a(n)=a$ for $n \geq n_{0}$. For each $n$ let the $\pi_{i, n}$ be ordered so that

$$
\Pi_{H, n} \pi_{i, n}=0 \text { for } j<i .
$$

Then

$$
\Pi_{H_{1, n}} \mu=\mu_{1, n}=\sum_{j} \Pi_{H_{1, n}} \mu_{j, n+1}
$$

Now $\Pi_{H} \mu_{1, n} \mu_{j, n+1}=\mu_{j, n+1}$ or 0 . Pick the smallest $j$ so that it is not 0 and apply $\Pi_{H_{j, n+1}}$ to (6). Using the fact that $H_{j, n+1}$ is normal in $A_{n}$ as well as $A_{n+1}(5)$ gives $\mu_{1, n}=\mu_{j, n+1}$. By repeating this process, and also reordering the $\prod_{j, n}$, we obtain that

$$
\mu_{j, n}=\mu_{j, n+1} \quad\left(1 \leq j \leq a ; n \geq n_{0}\right)
$$

Now let $\mu_{j}=\mu_{j, n^{*}}$. Then, for $n \geq n_{0}, \mu_{j} \in F\left(A_{n}\right)$. Since $\bigcup_{n_{0}}^{\infty} A_{n}$ is dense in $G$ we must then have that $\mu_{j} \in M^{Z}(G)$ and so $\mu_{j} \in F(G)$. But the $\mu_{j}$ are mutually singular and $\mu$ is irreducible so that $a=1$. Hence $\mu$ itself can be written, for $n \geq n_{0}$, as $\mu=\mu_{1, n}$. Thus $\mu<<\mathbb{M}_{1, n}$ and so $\mu$ is carried by $H_{1, n}$ Z: $L$. But since $L$ is the support group of $\mu$ and $H_{1, n}$ is closed we must have $H_{1, n}=L$. Thus $\mu$, being absolutely continuous with respect to the Haar measure of $L$, a closed normal subgroup of $G$, is canonical with respect to $G$

5. Norms of canonical measures. It is known [7, Theorem 3.7.2] that if $\mu$ is an idempotent measure on an abelian group and $\|\mu\|>1$ then $\|\mu\|>\sqrt{ } 5 / 2$. This section contains a generalization of this to canonical measures on compact groups.

Lemma 5.1. Let $P=\Sigma a_{a} X_{a}$ be a polynomial on $G$. Suppose $a_{a} \geq 0$ and $\|P\|_{\infty}=P(e)=1$. If

$$
\left|P\left(g_{i}\right)-z_{i}\right| \leq \delta_{i} \quad(1 \leq i \leq p)
$$

where $\left|z_{i}\right|=1$ then

$$
\left|P\left(g_{1} \cdots g_{p}\right)-\prod_{1}^{p} z_{i}\right| \leq\left(\sum \delta_{i}^{1 / 2}\right)^{2} .
$$


Proof. The lemma needs only to be proved for $p=2$ as the general case then easily follows by induction. If $T_{a}$ is a representation affording $X_{a}$ we can assume $T_{a}\left(g_{1}\right)$ is a diagonal unitary matrix with diagonal entries $b_{a, i}(1 \leq i$ $\left.\leq d_{a}\right)$ and $T_{a}\left(g_{2}\right)$ is a unitary matrix with diagonal entries $c_{a_{3} i}$. Then

$$
\begin{aligned}
\chi_{a}\left(g_{1}\right) & =\sum_{i} b_{a, i}, \quad \chi_{a}\left(g_{2}\right)=\sum_{i} c_{a, i} \text { and } \\
\chi_{a}\left(g_{1} g_{2}\right) & =\sum_{i} b_{a, i} c_{a, i} \cdot
\end{aligned}
$$

Since $\Sigma_{a_{a}} d(\alpha)=1,(2)$ follows directly from (1).

Lemma 5.2. Let $Q=\Sigma_{E} d(a) \chi_{\alpha}$ be a central idempotent polynomial on $G$. If $\|Q\|_{1}>1$ then

$$
\|Q\|_{1}>1+1 / 300
$$

Proof. Suppose

$$
\|Q\|_{1} \leq 1+1 / 300
$$

we will show $\|Q\|_{1}=1$. It can be assumed that $G$ is the support group of $Q$. Write $|Q|^{2}=\Sigma a_{a} X_{a}$ and $|Q|^{4}=\Sigma b_{a} X_{a}$. The $a_{a}$ and $b_{a}$ are nonnegative integers and

$$
a_{a}=\int|Q|^{2} \bar{\chi}_{a} \leq M d(\alpha), \quad b_{a}=\int|Q|^{4} \bar{\chi}_{a} \leq M^{3} d(\alpha)
$$

where

$$
M=\sum_{E} d^{2}(a)=\|Q\|_{\infty}=Q(e)=\|Q\|_{2}^{2} .
$$

Also, by Hölder's inequality,

$$
\sum a_{a} b_{a}=\int|Q|^{6} \geq M^{5}\|Q\|_{1}^{-4} .
$$

Define $A_{1}(g)=M^{-4} \Sigma_{b_{a}}\left(1-a_{a}(M d(a))^{-1}\right) \chi_{a}(g)$ and

$$
A_{2}(g)=M^{-2} \sum a_{a}\left(1-b_{a}\left(M^{3} d(a)\right)^{-1}\right) \chi_{a}(g) .
$$

It follows from (4)-(7) that

$$
\left\|A_{i}\right\|_{\infty}=A_{i}(e) \leq 1-\|Q\|_{1}^{-4}<1 / 60 \quad(i=1,2) .
$$

Thus

$$
0 \leq|Q|^{2} / M^{2}-|Q|^{4} / M^{4}=A_{2}-A_{1}<1 / 30 .
$$


Hence, for all $g$, either

$$
|Q(g)|<M / 5 \text { or }
$$

$$
|Q(g)|>4 M / 5 \text {. }
$$

If $g_{1}$ and $g_{2}$ satisfy (11) then applying Lemma 5.1 to $P=Q / M$ shows $\left|Q\left(g_{1} g_{2}\right)\right|$ $\geq M / S$ so that $g_{1} g_{2}$ must also satisfy (11). Hence the $g$ for which (11) holds form a closed normal subgroup $H$. Since (10) holds on $H^{c}$ we have from (4) and (6) that

$$
\frac{4}{5} \int_{H^{c}}|Q| \leq \int_{H^{c}}|Q|\left(1-\frac{|Q|}{M}\right) \leq \int|Q|\left(1-\frac{|Q|}{M}\right) \leq \frac{1}{300} .
$$

It follows from (12) and Lemma 4.2 that the idempotent measure $Q)_{G}$ is carried on $H$; that is $Q$ vanishes on $H^{c}$. It was assumed however that $G$ is the support group of $Q$. Hence (11) holds for all $g$. But then $4 M / 5 \leq\|Q\|_{1} \leq 1+1 / 300$ so that $M=1$. This then implies that $Q$ is just a character of degree 1 and the proof is complete.

Theorem 5.3. If $\mu$ is a canonical measure and $\|\mu\|>1$ then $\|\mu\|>1+$ $1 / 700$.

Proof. If $\mu$ is reducible then $\|\mu\| \geq 2$. An irreducible canonical measure is absolutely continuous with respect to $\mathbb{N}_{H}$ for some closed normal subgroup H. Also $\|\mu\| \geq|\hat{\mu}(a)|$. Thus we can assume that $\mu$ is given by a polynomial $Q=\Sigma( \pm 1) d(\alpha) \chi_{a}$. Suppose $\|Q\|_{1} \leq 1+1 / 700$. If either $\pm Q$ is idempotent then $\|Q\|_{1}=1$ by Lemma 5.2. Otherwise $Q * Q$ and $(Q * Q \pm Q) / 2$ are nonzero idempotents with norms less than $1+1 / 300$ and so, by Lemma 5.2, they all have norm 1. It is easily seen from this that $Q=\gamma_{1}-\gamma_{2}$ where $\gamma_{1}$ and $\gamma_{2}$ are distinct characters of degree 1 with $\gamma_{1}^{2}=\gamma_{2}^{2}$. It follows that $|Q|=0$ on a subgroup of index 2 and $|Q|=2$ on the complement of this subgroup. Thus $\|\mu\|=$ $\|Q\|_{1}=1$.

The estimates in 5.2 and 5.3 can easily be improved. It would be interesting to know the best ones. A special case of 5.2 is that if $\left\|d(a) \chi_{a}\right\|_{1}>1$ then $\left\|d(a) \chi_{a}\right\|_{1}>1+1 / 300$, whenever $\chi_{a}$ is an irreducible character. It should be noted that a group can be constructed having a sequence $\{a\} \subseteq \Gamma$ with $d(\alpha) \rightarrow$ $\infty$ and $\left\|d(\alpha) \chi_{a}\right\|_{1}=1$ and having another sequence $\{\beta\}$ with $d(\beta) \rightarrow \infty$ and $1<\left\|d(\beta) \chi_{\beta}\right\|_{1} \leq 2$.

6. $F(G)$ for special $G$. This section contains some rather technical results which are necessary for the proof of Theorem 2.2. We will first prove a special case of that theorem. 
Theorem 6.1. Let $G_{i}(1 \leq i \leq n)$ be compact groups satisfying conditions I and II and let $G=\Pi G_{i}$. Then every measure in $F(G)$ is canonical.

Proof. The proof will be by induction on $\|\mu\|$. If $\|\mu\|=1$ then it is known [3, The orem 2.1.4] that $\mu$ is canonical. Suppose that for such $G$ every measure in $F(G)$ with norm less than $A$ is canonical and let $\|\mu\|<A+1$. We can assume $\mu$ is irreducible.

Say that $\mu$ is of bounded representation type (b.r.t.) if there is $M<\infty$ such that $\hat{\mu}(\alpha)=0$ whenever $d(\alpha)>M$. If $\mu$ is not of b.r.t. then, for some $j \neq 0, E_{j}(\mu)$ contains a sequence $\{\alpha\}$ with $d(\alpha) \rightarrow \infty$. Now $\alpha$ can be written as $\alpha=a_{1} \alpha_{2}$ $\ldots a_{n}$ where $a_{i} \in \Gamma\left(G_{i}\right)$ and $d(\alpha)=d\left(\alpha_{1}\right) \ldots d\left(a_{n}\right)$. For some $i$, say $i=1$, $d\left(a_{1}\right) \rightarrow \infty$. Since $G_{1}$ satisfies condition $I_{1} \Psi_{\alpha_{1}} \rightarrow 0$ off $Z_{1}=Z\left(G_{1}\right)$. Thus since

$$
j=\hat{\mu}(a)=\int \bar{\Psi}_{a} d \mu
$$

we having, using the Lebesgue dominated convergence theorem, that $|\mu|\left(Z_{1} \times\right.$ $\left.\Pi_{2}^{n} G_{i}\right) \neq 0$. Since $\mu$ is irreducible it follows from Theorem 3.2 that $L \cap\left(Z_{1} \times\right.$ $\left.\Pi_{2}^{n} G_{i}\right)$ is open in $L$ ( $L$ is the support group of $\mu$ ). Thus $L \subset K_{1} \times \Pi_{2}^{n} G_{i}=G_{i}^{*}$ where $K_{1}$ is a finite normal extension of $Z_{1}$.

There are now three possibilities:

(a) $\mu$ is irreducible but not of b.r.t. on $G^{*}$.

(b) $\mu$ is irreducible and of b.r.t. on $G^{*}$.

(c) $\mu$ is reducible on $G^{*}$.

For $\beta \in \Gamma\left(K_{1}\right), d(\beta) \leq\left[K_{1}: Z_{1}\right]^{1 / 2}<\infty$. Thus in case (a) we can repeat the above process and obtain that $L \subset K_{1} \times K_{2} \times \Pi_{3}^{n} G_{i}$ where $K_{2}$ is a finite normal extension of $Z\left(G_{2}\right)$. With respect to this new group, one of the above cases holds.

After a finite number of such steps we obtain that, for some rearrangement of the $G_{i}$ and some $m, L \subset H_{1} \times H_{2}=G^{*}$ where $H_{1}$ is a finite normal extension of $Z\left(\Pi_{1}^{m} G_{i}\right)$ and $H_{2}=\Pi_{m+1}^{n} G_{i}$ and either (b) or (c) holds for this $G^{*}$. Since $H_{1}$ has an open center it satisfies both conditions I and II. $G^{*}$ also satisfies condition II because it is the product of groups that do.

If case (b) holds then by Theorem 6 of [5] and Lemma 1.1 we have that $\mu$ is canonical on $G^{*}$. (Theorem 6 of $[5]$ is stated for $J$ but it applies equally well to $F$.) If case (c) holds then $\mu$ is a sum of measures in $F\left(G^{*}\right)$ each of norm less then $A$ and so by the induction hypothesis $\mu$ is canonical on $G^{*}$. Lemma 4.6 then gives that $\mu$ is canonical on $G$.

Lemma 6.2. Let $G_{i}(1 \leq i<\infty)$ be compact groups satisfying conditions I and II and let $G=\Pi G_{i}$. Suppose $\mu \in J(G)$ satisfies

$$
\alpha \in E(\mu) \text { if and only if }\left\|\left(\Psi_{\alpha}-1\right) \mu\right\|<1 / 300 \text {. }
$$


Tben $\mu$ is canonical; in fact $\mu=\pi_{H}$ for some closed normal subgroup $H$.

Remark. For abelian groups this is obvious. The problem in the nonabelian case is that $\Psi_{\alpha} \mu$ need not belong to $F(G)$. It seems likely that the restriction on $G$ can be lifted but I have been unable to do so. It does hold, however, if $G$ is totally disconnected.

Proof. Let $\pi_{r}$ be the Haar measure of $\Pi_{r+1}^{\infty} G_{i} \subset G$ and let $\mu_{r}=\mu * \pi_{r}$. Then $\mu_{r} \rightarrow \mu$ weakly and $\mu_{r}$ can be considered as an element of $J\left(\Pi_{1}^{r} G_{i}\right)$. (2) still holds for $\mu_{r}$ which is canonical by Theorem 6.1. We will show that $\left\|\mu_{r}\right\|=1$ for all $r$. It then follows that $\|\mu\|=1$ so that, by [3, Theorem 2.1.4], $\mu$ is canonical. Since $1 \in E(\mu)$, where 1 is the trivial representation, $\mu$ is a Haar measure.

Since $\mu_{r}$ is canonical it can be written as $\mu_{r}=\Sigma_{1}^{s} \nu_{j}$ where $\nu_{j} \in F(G)$ and $\nu_{j}<<\lambda_{j}$, the Haar measure of a closed normal subgroup $H_{j}$. We show first that $s=1$. We can assume the $\lambda_{j}$ are mutually singular and that $\Pi_{H_{1}} \lambda_{j}=0$ for $j>1$. Now if $a \in E\left(\mu_{r}\right)$ then

$$
\left\|\left(\Psi_{a}-1\right)_{j}\right\| \leq\left\|\left(\Psi_{a}-1\right) \mu_{r}\right\|<1 / 300 .
$$

Fix $\gamma \in E\left(\nu_{j}\right)$ then by (3)

$$
\left|\int\left(\bar{\Psi}_{a-1}\right) \bar{\Psi}_{\gamma} d \nu_{j}\right|=\left|\int \bar{\Psi}_{a} \bar{\Psi}_{\gamma} d \nu_{j}-\hat{\nu}_{j}(\gamma)\right|<1 / 300 .
$$

Since $\hat{\nu}_{j}(\gamma)$ is a nonzero integer, (4) implies that the decomposition of $\alpha \otimes \gamma$ contains an element of $E\left(\nu_{j}\right)$. Thus $E\left(\mu_{r}\right) \subset \bar{\gamma} E\left(\nu_{j}\right)$ where $\bar{\gamma}$ is the representation conjugate to $\gamma$ and $\bar{\gamma} E\left(\nu_{j}\right)$ consists of the $\beta \in \Gamma$ that appear in the decomposition of $\bar{\gamma} \otimes \theta$ for some $\theta \in E\left(\nu_{j}\right)$. Now $\nu_{j}<<\lambda_{j}$ which implies that $E\left(\nu_{j}\right)$ is the union of finitely many hypercosets of $H_{j}^{\perp}$. Thus $E\left(\mu_{r}\right)$ is contained in the union of finitely many hypercosets of $H_{j}^{\perp}$ so that there is $\omega_{j} \in J(G)$ with $\omega_{j}<<\lambda_{j}$ and $\mu_{r} * \omega_{j}=\mu_{r} \cdot$ Let $j>1$ and apply $\Pi_{H_{1}}$ to this last equality. Since $\Pi_{H_{1}} \lambda_{j}$ $=0$ it follows that $0=\Pi_{H_{1}} \mu_{r}=\lambda_{1}$. This is a contradiction so that $s=1$ and $\mu_{r}=\lambda_{1}$.

We can thus write $\mu_{r}=Q \lambda_{1}$ where $Q$ is a central idempotent polynomial on $H_{1}$ and also $Q=\left.\Sigma_{E} a_{a} X_{a}\right|_{H_{1}}$ where $a_{a}>0$ and $E \subset E\left(\mu_{r}\right)$. It follows from (2) that

$$
\int|(Q / Q(e)-1) Q| d \lambda_{1} \leq \sum_{E} \frac{a_{a} d_{(a)}\left\|\left(\Psi_{a}-1\right) \mu_{r}\right\|}{\Sigma_{E} a_{a^{d}(a)}}<\frac{1}{300} .
$$

Thus since $\left.\int Q\right|^{2} d \lambda_{1}=Q(e)$ it follows from (5) that

$$
\left\|\mu_{r}\right\|=\int|Q| d \lambda_{1}<1+1 / 300
$$


so that, by Lemma 5.2, $\left\|\mu_{r}\right\|=1$ and the proof is complete.

Lemma 6.3. Let $p$ be a positive integer and $M\langle\infty$. There is $\delta=\delta(p, M)>$ 0 sucb that if $G$ is as in 6.2 and $\mu \in F(G)$ satisfies the following:

(a) $\|\mu\| \leq M$.

(b) There is a normal Borel subgroup $T$ of $G$ with $|\mu|\left(T^{c}\right)=0$.

(c) There are Borel bomomorphisms $f_{1}, f_{2}, \ldots, f_{p}$ of $T$ into the unit circle with $f_{1} \equiv 1, f_{i} \mu \neq f_{j} \mu$ for $i \neq j$ and for eacb $i$ there is an irreducible sequence $\{a\} \subset E(\mu)$ with $\Psi_{a} \rightarrow f_{i}$ pointwise on $T$.

(d) $a \in E(\mu)$ if and only if $\left\|\left(\Psi_{a}-\bar{f}_{i}\right) \mu\right\|<\delta$ for some $i$, then $\omega=f_{1} \mu * f_{2} \mu * \ldots * f_{p} \mu=A \mathbb{M}_{H}$ for some integer $A \neq 0$ and some closed normal subgroup $H$.

Proof. $\delta$ is chosen so that

$$
p \delta^{1 / 2} M^{p-1}(p M+2)<1 / 300 \text {. }
$$

Clearly each $f_{2} \mu \in F(G)$ so that $\omega \in F(G)$. Also, by (c),

$$
\int f_{i} d \mu=\lim \int \bar{\Psi}_{a} d \mu \neq 0 \text { so that } \hat{\omega}(1)=\prod\left(f_{t} \mu\right) \hat{(1)} \neq 0 .
$$

Let $\beta$ be a fixed element of $E(\omega)$. Then $\beta \in E\left(f_{i} \mu\right)$ for all $i$ so that, by using (c), $\lim \int \bar{\Psi}_{\beta} \bar{\Psi}_{\alpha} d \mu=\int \bar{\Psi}_{\beta} f_{2} d \mu \neq 0$. Since $\{a\}$ is an irreducible sequence we must have $\beta \otimes a$ irreducible eventually and $\beta \alpha \in E(\mu)$. Thus (d) implies that

$$
\left\|\left(\Psi_{\beta} \Psi_{a}-\bar{f}_{j}\right) \mu\right\|<\delta \quad \text { for some } j .
$$

Since $\mu$ is carried by $T$ it follows from (c) that

$$
\left\|\left(\Psi_{\beta}-f_{i} \bar{f}_{j}\right) \mu\right\|=\left\|\left(\Psi_{\beta} \bar{f}_{i}-\bar{f}_{j}\right) \mu\right\| \leq \delta .
$$

Now since $f_{1} \equiv 1, E(\omega) \subset E(\mu)$ so that there is $k$ with

$$
\left\|\left(\Psi_{\beta}-\bar{f}_{k}\right) \mu\right\|<\delta \text {. }
$$

We will show for such a $k$ that

$$
f_{k} \omega=\omega \text {. }
$$

Since $\bar{f}_{k} \mu$ and $f_{i} \bar{f}_{j} \mu$ are both in $F(G)$ it follows from (9) and (10) that (since $\delta<1 / 2) \bar{f}_{k} \mu=f_{i} \bar{f}_{j} \mu$. Hence for this $k$ and all $i$ there is $j$ with $f_{i} f_{k} \mu=f_{j} \mu$. Since $\left\{f_{i} \mu\right\}$ are distinct it follows that

$$
\left\{f_{i} f_{k} \mu: 1 \leq i \leq p\right\}=\left\{f_{i} \mu: 1 \leq i \leq p\right\} .
$$

Now the $f_{i} \mu$ are central measures so that if $\sigma$ is a permutation of $(1,2, \cdots, p)$ then 


$$
\omega=f_{\sigma(1)} \mu * \cdots * f_{\sigma(p)}{ }^{\mu} .
$$

Let $\lambda=\mu \times \mu \times \cdots \times \mu$ on $G \times G \times \cdots \times G$. Then, for $\gamma \in \Gamma$, it follows from (12) and (13) that for some permutation $\sigma$

$$
\begin{aligned}
\left(f_{k} \omega\right) \hat{(y)} & =\int \bar{\Psi}_{\gamma} f_{k} d \omega \\
& =\int \cdots \int \bar{\Psi}_{\gamma}\left(g_{1} \cdots g_{p}\right) f_{k}\left(g_{1} \cdots g_{p}\right) \prod_{1}^{p} f_{i}\left(g_{i}\right) d \lambda \\
& =\int \cdots \int \bar{\Psi}_{\gamma}\left(g_{1} \cdots g_{p}\right) \prod f_{\sigma(i)}\left(g_{i}\right) d \lambda=\int \bar{\Psi}_{\gamma} d \omega=\hat{\omega}(\gamma) .
\end{aligned}
$$

(11) then follows from (14).

Now let $R=\left\{g \in T:\left|\Psi_{\beta}(g)-\bar{f}_{k}(g)\right|<\delta^{1 / 2}\right\}$. It follows from (10) that

$$
|\mu|\left(R^{c}\right) \leq \delta^{1 / 2}
$$

It also follows from Lemma 5.1 that if $g_{i} \in R(1 \leq i \leq p)$

$$
\left|\Psi_{\beta}\left(g_{1} \cdots g_{p}\right)-\bar{f}_{k}\left(g_{1} \cdots g_{p}\right)\right|<p^{2} \delta^{1 / 2} .
$$

Then by (7), (11), (15), (16) and (a)

$$
\begin{aligned}
& \left\|\left(\Psi_{\beta}-1\right) \omega\right\|=\left\|\left(\Psi_{\beta}-\bar{f}_{k}\right) \omega\right\| \\
& \quad \leq \int \cdots \int\left|\Psi_{\beta}\left(g_{1} \cdots g_{p}\right)-\bar{f}_{k}\left(g_{1} \cdots g_{p}\right)\right| d(|\mu| \times \cdots \times|\mu|) \\
& \quad \leq p^{2} \delta^{1 / 2} M^{p}+2 p M^{p-1} \delta^{1 / 2}<1 / 300 .
\end{aligned}
$$

The last inequality is obtained by integrating over $S=R \times \cdots \times R$ and $S^{c}$ separately.

(17) holds for all $\beta \in E(\omega)$. Lemma 6.2 can then be applied to show that $\omega / \hat{\omega}(1)$ is a Haar measure.

7. Some more technical lemmas. The main purpose of this section is to prove Lemma 7.6.

Lemma 7.1. Let $H$ be a closed normal subgroup of G. Suppose $\{a\}$ is a sequence in $\Gamma$ sucb that

$$
\left|\Psi_{a}(g)\right| \rightarrow 1 \text { a.e. }\left(\pi_{H}\right)
$$

Then, for a large enough, $\left|\Psi_{a}(g)\right| \equiv 1$ on $H$.

Proof. Since $H$ is closed and normal we can write $\left.\chi_{a}\right|_{H}=a \Sigma_{1}^{p} \chi_{\beta_{i}}$ where $\beta_{i} \in \Gamma(H)$ and $a p d\left(\beta_{i}\right)=d(\alpha)$ for all $i$. Then 


$$
\int_{H}\left|\Psi_{a}\right|^{2} d \pi_{H}=a^{2} p(d(\alpha))^{-2}=\left(p d^{2}\left(\beta_{1}\right)\right)^{-1}
$$

By $(1)$ and the Lebesgue dominated convergence theorem $\left(p d^{2}\left(\beta_{1}\right)\right)^{-1} \rightarrow 1$. Thus eventually $p=d\left(\beta_{1}\right)=1$. That is $\left.\chi_{\alpha}\right|_{H}=d(\alpha) \gamma$ where $\gamma \in \Gamma(H)$ and $d(\gamma)=1$ so that $\left|\Psi_{a}\right| \equiv 1$ on $H$.

Lemma 7.2. Let $H$ and $K$ be closed normal subgroups of $G$ with $H$ open in $K$. Let $f$ be the characteristic function of $H$. Suppose $\{a\}$ is a sequence in $\Gamma$ sucb that

$$
\left|\Psi_{a}(g)\right| \rightarrow f(g) \text { a.e. }\left(\pi_{k}\right) .
$$

Then, for a large enough, $\left|\Psi_{\alpha}\right| \equiv f$ on $K$.

Proof. From Lemma 7.1 we can assume $\left|\Psi_{a}\right| \equiv 1$ on. $H$. Write $\left.\chi_{a}\right|_{K}=$ $a \Sigma_{1}^{p} \chi_{\beta_{i}}$ where $\beta_{i} \in \Gamma(K)$. It follows as in the previous proof that

$$
\left(p d^{2}\left(\beta_{1}\right)\right)^{-1}=\int_{K}\left|\Psi_{\alpha}\right|^{2} d \pi_{K}=\pi_{K}(H)+o(1) .
$$

Thus eventually $\left(p d^{2}\left(\beta_{1}\right)\right)^{-1}=\pi_{K}(H)$ and so $\int_{K-H}\left|\Psi_{a}\right|^{2} d \pi_{K}=0$; that is $\Psi_{\alpha}=$ 0 on $K-H$.

Lemma 7.3. Let $H$ and $K$ be closed normal subgroups of $G$ with $H$ open in $K$. Let $E \subset \Gamma$ be sucb that $\left\{\left.\Psi_{a}\right|_{H}: a \in E\right\}$ is finite. Then $\left\{\left.\Psi_{a}\right|_{K}: a \in E\right\}$ is also finite.

Proof. If the lemma is false there is a sequence $\{a\} \subset \Gamma$ such that $\Psi_{a} l_{K}$ are distinct and $\left.\Psi_{a}\right|_{H}$ are all the same. By Lemma $4.4 \Psi_{a_{0}} \pi_{H}$, being a weak limit point of $\left\{\Psi_{a} \pi_{H}\right\}$, is singular to $\pi_{K}$. But $\pi_{H}<<\pi_{K}$ which gives a contradiction.

The following lemma was proved by Amemiya and Itô [1] although not stated in this form:

Lemma 7.4. Let $\mu$ and $\nu$ be nonzero regular Borel measures on some space sucb that $f_{n} \mu \rightarrow \nu$ weakly where $\left\|f_{n}\right\|_{\infty} \leq 1$. Then given $k<\|\nu\| /\|\mu\| \leq 1$ there is $N$ sucb that

$$
\left\|\left(f_{n}-f_{m}\right) \mu\right\|<2\|\mu\|\left(1-k+\left(1-k^{2}\right)^{1 / 2}\right) \text { for } n, m \geq k_{0}
$$

Lemma 7.5. If, in the above, $\|\mu\|=\|\nu\|$ then $f_{n} \mu \rightarrow \nu$ in norm.

Proof. Letting $k$ be close to 1 in (5) shows that $\left\{f_{n} \mu\right\}$ is a Cauchy sequence.

It also follows immediately from Lemma 7.4 that if $1 \leq A \leq\|\nu\| \leq\|\mu\|<A+$ $1 / 100 A$ then

$$
\left\|\left(f_{n}-f_{m}\right) \mu\right\|<1 / 4 \text { for large } n, m \text {. }
$$


Lemma 7.6. Let $G_{i}(1 \leq i<\infty)$ be compact groups satisfying condition II and let $G=\Pi G_{i}$. Let $\mu \in F(G)$ bave support group $L$. If $\mu$ satisfies the following two conditions then $\mu$ is canonical.

(a) There is an integer $N$ so that if $P$ is a normal Borel subgroup of $G$ and $\Pi_{1}^{N} G_{i}^{\prime} \subset P\left(G_{i}^{\prime}\right.$ is the commutator subgroup of $\left.G_{i}\right)$ then $\Pi_{P} \mu \neq 0$ implies $P \cap L$ is open in $L$.

(b) For each $i$ there is $M_{i}<\infty$ so that if $\alpha \in E(\mu)$ and $\alpha=a_{1} \cdots a_{n}$ where $\alpha_{i} \in \Gamma\left(G_{i}\right)$ then $d\left(\alpha_{i}\right)<M_{i}$.

Proof. The proof is by induction on $\|\mu\|$. Assume it is true for $1 \leq\|\mu\|<A$ and let $A \leq\|\mu\|<A+1 / 100 A$. We will show that $T=\left\{\left.\Psi_{a}\right|_{L}: a \in E(\mu)\right\}$ is finite; $\mu$ is then canonical by Lemma 4.1 .

We will assume $T$ is infinite in order to obtain a contradiction. By using a diagonal process and the fact that each $G_{i}$ satisfies condition II it follows from (b) that there is a sequence $\left\{a_{n}\right\} \subset E(\mu)$ such that

(i) $a_{n}=a_{n, 1} a_{n, 2} \cdots$ where $a_{n, i} \in \Gamma\left(G_{i}\right)$,

(ii) there are $\beta_{i} \in \Gamma\left(G_{i}\right)$ such that $\alpha_{n, i}=\beta_{i} \gamma_{n, i}$ for $n \geq n(i)$ where $\gamma_{n, i}$ $\epsilon \Gamma\left(G_{i}\right)$ and $d\left(\gamma_{n, i}\right)=1$, and

(iii) $\left.\Psi_{a}\right|_{L}$ are distinct.

By taking a subsequence of $\left\{a_{n}\right\}$ we can write

$$
a_{n}=\beta_{1} \cdots \beta_{n} \gamma_{n} \lambda_{n}
$$

where $d\left(\gamma_{n}\right)=1$ and $\lambda_{n} \in \Gamma\left(\Pi_{n+1}^{\infty} G_{i}\right)$.

We will first show that there is $i_{0}>N$ such that

$$
\left|\Psi_{\beta_{i}}\right|_{L} \equiv 1 \quad \text { for } i \geq i_{0} .
$$

Let $P=\left\{g: \lim _{i \rightarrow \infty}\left|\Psi_{\beta_{i}}(g)\right|=1\right\} . P$ is clearly a normal Borel subgroup and by (7) $\Psi_{\alpha_{n}} \rightarrow 0$ on $P^{c}$ as $n \rightarrow \infty$. Since $\hat{\mu}\left(\alpha_{n}\right) \neq 0$ it follows that $|\mu|(P) \neq 0$.

Thus since $\Pi_{1}^{N} G_{i}^{\prime} \subset P$ it follows from (a) that $P \cap L$ is open in $L$. In particular $P \cap L$ is closed so that by Lemma 7.1 there is $i_{0}>N$ such that $\left|\Psi_{\beta_{i}}\right| \equiv 1$ on $P \cap L$ for $i \geq i_{0}$. We will show $P \cap L=L$.

If $P \cap L \neq L$ then, by Lemma 4.2,

$$
|\mu|(L-P \cap L) \geq 1 / 2 \text {. }
$$

Also $\lim _{p \rightarrow \infty}\left|\Psi_{\beta_{i_{0}}} \ldots \Psi_{\beta_{p}}\right|=0$ on $L-P \cap L$ so that if $p$ is large enough it follows from Lemma 7.2 that

$$
\Psi_{\beta_{i_{0}}} \ldots \Psi_{\beta_{p}} \equiv 0 \text { on } P \cap L \text {. }
$$

Let $\theta=\beta_{i} \cdots \beta_{p}$ and let $K=\Pi_{1}^{p} G_{i}^{\prime}$. Define $\phi=\bar{\Psi}_{\theta} \mu * \pi_{K}$. It is easily seen that $\phi \neq 0, \phi \in F(G)$ and 


$$
\sigma_{n}=\beta_{p+1} \cdots \beta_{n} \gamma_{n} \lambda_{n} \in E(\phi) \text { for } n>p
$$

Also since $\Psi_{\theta}$ vanishes on $L-(L \cap P)$ it follows from (9) that $\|\phi\| \leq\|\mu\|-1 / 2$ $<A$. Also $\phi$ satisfies (a) and (b). (b) is easy. To see (a) (with $p$ in place of $N)$ suppose $K \subset Q$ and $\Pi_{Q} \phi \neq 0$. Then $\Pi_{Q} \mu \neq 0$ so that $Q \cap L$ is open in $L$. Now the support group $L^{*}=L(\phi) \subset(L \cap P) K$. Thus $L^{*} Q \subset L Q . Q$ is open in $L Q$ and thus is also open in $L^{*} Q$; that is $L^{*} \cap Q$ is open in $L^{*}$. It follows from the induction hypothesis that $\phi$ is canonical.

Since $\phi$ is canonical it follows that for some closed normal subgroup $H$ with $\Pi_{H} \phi \neq 0$ there is an infinite set $I$ such that $\sigma_{n} \in \sigma_{n_{0}} H^{\perp}$ for all $n \in I$; that is $\left.\Psi_{\sigma}\right|_{H}$ are the same for $n \in I$. Since $\phi * M_{K}=\phi$ we can assume $K \subset H$ so that $H^{n} \cap L$ is open in $L$. By Lemma 7.3 this implies that $\left\{\left.\Psi_{\sigma_{n}}\right|_{L}: n \in I\right\}$ is finite. But $a_{n}=\theta \sigma_{n}$ so that we have a contradiction to (iii). Hence $P \cap L=L$ and (8) is proved.

Now let $\theta_{n}=\beta_{i_{0}} \cdots \beta_{n} \gamma_{n} \lambda_{n}$. A subsequence of $\left\{\bar{\Psi}_{\theta_{n}} \mu\right\}$ converges weakly to some $\omega$. Now $\omega \neq 0$ and $\omega \in F(G)$; this is because the ${ }^{n} \Psi_{\beta_{i}}$ are multiplicative on $L$ for $i \geq i_{0}$ and because $\left\{\gamma_{n} \lambda_{n}\right\}$ is an irreducible sequence. Now $\omega$ clearly satisfies (b). To see (a) suppose $\Pi_{p} \omega \neq 0$; since $\omega<<\mu$ it follows that $\Pi_{p} \mu \neq$ 0 . Hence if $\Pi_{1}^{N} G_{i}^{\prime} \subset P$ we have $P \cap L$ open in $L$. But $L(\omega) \subset L$ so that $P \cap L(\omega)$ is open in $L(\omega)$. The remainder of the proof is divided into two cases.

Case 1. $\|\omega\|<A$. Then $\omega$ is canonical by the induction hypothesis. Let $H$ be a closed normal subgroup of $G$ such that $0 \neq \Pi_{H} \omega<<\Pi_{H}$. Since $\left.\bar{\Psi}_{\theta} \mu\right|_{H}$ $\left.\rightarrow \omega\right|_{H}$ weakly it follows from Lemma 4.4 that $\left\{\left.\Psi_{\theta_{n}}\right|_{H}\right\}$ is finite. Now $\Psi_{\theta_{n}}{ }_{n} \equiv 1$ on $\Pi_{1}^{N} G_{i}^{\prime}$ so that $\left\{\left.\Psi_{\theta}\right|_{s}\right\}$ is finite where $S=H \cdot \Pi_{1}^{N} G_{i}^{\prime}$. Also $\Pi_{s^{\mu}} \neq 0$ so that $S \cap L$ is open in $L$. It follows from Lemma 7.3 that $\left\{\left.\Psi_{\theta_{n}}\right|_{L}\right\}$ is finite and since $a_{n}=\beta_{1} \cdots \beta_{i_{0}-1} \theta_{n}$ this contradicts (iii).

Case 2. $\|\omega\| \geq A$. It follows here from the remark (6) after Lemma 7.5 that, if $n, m$ are large enough,

$$
\left\|\left(\bar{\Psi}_{\theta_{n}}-\bar{\Psi}_{\theta_{m}}\right) \mu\right\|<1 / 4 .
$$

Fixing $n$ and letting $m \rightarrow \infty$ in (12) then gives

$$
\left\|\bar{\Psi}_{\theta_{n}} \mu-\omega\right\| \leq 1 / 4
$$

and

$$
\left\|\bar{\Psi}_{\theta_{n}} \mu\right\| \geq\|\omega\|-1 / 4 \geq A-1 / 4 \text {. }
$$

By (13) and (14), using that $\left|\Psi_{\theta_{n}}\right| \leq 1$, 


$$
\begin{aligned}
\left\|\mu-\Psi_{\theta_{n}} \omega\right\| & \leq\left\|\left(1-\left|\Psi_{\theta_{n}}\right|^{2}\right) \mu\right\|+\left\|\left|\Psi_{\theta_{n}}\right|^{2} \mu-\Psi_{\theta_{n}} \omega\right\| \\
& \leq 2\left[\|\mu\|-\left\|\bar{\Psi}_{\theta_{n}} \mu\right\|\right]+\left\|\bar{\Psi}_{\theta_{n}} \mu-\omega\right\| \\
& \leq 3 / 4+1 / 50 A .
\end{aligned}
$$

Since $\omega$ is carried by $L$ it follows that (a subsequence of) $\left\{\Psi_{\theta_{n}} \omega\right\}$ converges weakly to some $\lambda \in F(G)$. (15) show s that $\|\mu-\lambda\|<1$ so that $\mu=\lambda$. Since $\|\lambda\|$ $\leq\|\omega\|$ we must then have that $\|\omega\|=\|\mu\|$ so that by Lemma $7.5 \bar{\Psi}_{\theta_{n}} \mu \rightarrow \omega$ in norm. It then follows that, for a subsequence,

$$
\left|\Psi_{\theta_{n}}\right| \rightarrow 1 \text { a.e. }(|\mu|) \text {. }
$$

Now (16) occurs on a normal Borel subgroup $S$ which contains $\Pi_{1}^{N} G_{i}^{\prime}$. Thus, by (a), $S \cap L$ is open in $L$. $S \cap L$ is then closed and carries $\mu$ so that $S \cap L=$ $L$. This gives, by Lemma 7.1, that

$$
\left|\Psi_{\theta_{n}}\right| \equiv 1 \text { on } L \text { for large } n \text {. }
$$

But then $\bar{\Psi}_{\theta} \mu \in F(G)$ so that, because of (12),

$$
\bar{\Psi}_{\theta_{n} \mu}=\bar{\Psi}_{\theta_{m} \mu}
$$

Finally (17) and (18) show that $\left.\Psi_{\theta}\right|_{L}=\left.\Psi_{\theta}\right|_{L}$ which implies that $\left.\Psi_{\alpha_{n}}\right|_{L}=$ $\left.\Psi_{a_{m}}\right|_{L}$ and this contradicts (iii).

\section{Proof of Theorem 2.2.}

Theorem 2.2. Let $G_{i}(i \in A)$ be compact groups satisfying conditions I and II and let $G=\Pi_{A} G_{i}$. Then every measure in $F(G)$ is canonical. any $A$.

Proposition 8.1. If the theorem is true for countable products it is true for

Proof. Let $\mu \in F(G)$. We can assume $\mu$ is irreducible and has support group $L$. If $\mu$ is not canonical then by Lemma 4.1 there is a sequence $\left\{a_{n}\right\} \subset E(\mu)$ such that $\left.\Psi_{a_{n}}\right|_{L}$ are distinct. There is a countable set $B C A$ such that $a_{n} \epsilon$ $\Gamma\left(\Pi_{B} G_{i}\right)$ for all $n$. Let $K=\Pi_{A-B} G_{i}$ and $\nu=\mu * \Pi_{K}$. Then $E(\nu)$ contains $\left\{a_{n}\right\}$ and $\nu$ is canonical since it can be considered as a measure on $\Pi_{B} G_{i}$. It is then easily seen that $\nu$ is irreducible so that $\left\{\left.\Psi_{a_{n}}\right|_{L(\nu)}\right\}$ is finite. But $L(\nu) \cap$ $L$ is open in $L$ so that $\left\{\left.\Psi_{a_{n}}\right|_{L}\right\}$ is finite by Lemma 7.3 which is a contradiction.

It remains to prove the theorem when $A$ is countable. The proof is by induction on $\|\mu\|$. Assume it is true for $\|\mu\|<C$ and let $\|\mu\|<C+1 / 100 C$. We can also assume that $\mu$ is irreducible. 
Proposition 8.2. We can assume $\mu$ satisfies condition (b) of Lemma 7.6 and that $1 \in E(\mu)$.

Proof. Suppose $E(\mu)$ contains a sequence $\{a\}$ where $a=a_{1} a_{2} \ldots$ with $a_{i}$ $\epsilon \Gamma\left(G_{i}\right)$ and suppose, for some $j$, that $d\left(a_{j}\right) \rightarrow \infty$ as $a \rightarrow \infty$. Since $\hat{\mu}(\alpha) \neq 0$ and $G_{j}$ satisfies condition I it follows that

$$
|\mu|\left(\prod_{i \neq j} G_{i} \times Z\left(G_{j}\right)\right) \neq 0 .
$$

By Theorem 3.2 we then have that $L=L(\mu) \subset \Pi_{i \neq j} G_{i} \times K_{j}$ where $K_{j}$ is a finite normal extension of $Z\left(G_{j}\right)$. Doing this for all such $j$ we obtain that $L \subset \Pi K_{i}=G^{*}$ where $K_{i}=G_{i}$ or $K_{i}$ is a finite normal extension of $Z\left(G_{i}\right)$. Now each $K_{i}$ satisfies conditions I and II and $\mu$ satisfies condition (b) with respect to $G^{*}$. If $\mu$ is canonical with respect to $G^{*}$ then by Lemma 4.7 it is canonical with respect to $G$. It can still be assumed that $\mu$ is irreducible; if $\mu$ is reducible as an element of $F\left(G^{*}\right)$ then it is canonical on $G^{*}$ by the induction hypothesis and then it is canonical on $G$.

Let $\alpha=a_{1} \cdots a_{N} \in E(\mu)$ and let $K=\Pi_{1}^{N} G_{i}^{\prime}$. Then $\bar{\Psi}_{a} \mu * M_{K}=\lambda \in F(G)$, $1 \in E(\lambda)$ and $\|\lambda\| \leq\|\mu\|$. Also $\lambda$ satisfies condition (b) since we can now assume that $\mu$ does. We will show that if $\lambda$ is canonical then $\mu$ is also. Suppose $\lambda=$ $\Sigma \nu_{j}$ where $\nu_{j}<<M_{H}$ for some closed normal subgroup $H_{j}$. Let $P$ be a normal Borel subgroup with $K \subset P$ and $\Pi_{P} \mu \neq 0$. Then since $\mu$ is irreducible $\Pi_{P} \mu=\mu$; also $\Pi_{P} \mathbb{M}_{K}=\pi_{K}$ since $K \subset P$. Thus $\Pi_{P} \lambda=\lambda$ so that $\Pi_{P} \pi_{H_{j}}=\pi_{H_{j}}$ for all $j$. This implies that $P \cap H_{j}$ is open in $H_{j} \cdot$ But $\Pi_{H} \mu \neq 0$ so that $H_{j} \cap L$ is open in $L$. Hence $P \cap L$ is open in $L$. By Lemma 7.6 then $\mu$ is also canonical. We can thus assume $\mu$, like $\lambda$, has $1 \in E(\mu)$.

The remainder of the proof involves using Lemma 6.3 to show that $\mu$ satisfies condition (a) of Lemma 7.6.

Call a sequence $\left\{a_{n}\right\} \subset E(\mu)$ an F-sequence if $a_{n}=a_{n, 1} a_{n, 2} \cdots$ with $\alpha_{n, i} \in \Gamma\left(G_{i}\right)$ and $d\left(a_{n, i}\right)=1$ for $i \leq n$. An $F$-sequence is an irreducible sequence so that a subsequence of $\left\{\bar{\Psi}_{a_{n}} \mu\right\}$ will converge weakly to some nonzero $\omega \in F(G)$. Let $B$ be the collection of all such $\omega . \mu \in B$ since $a_{n}=1$ is an F-sequence. Now if $\omega \in B$ and $\|\omega\|<C$ then $\omega$ is canonical by induction and $\mu$ is then canonical by Lemma 4.5. We can thus assume that $\omega \in B$ implies

$$
C \leq\|\omega\| \leq\|\mu\|<C+1 / 100 C .
$$

It then follows, for $\omega \in B$, that, as in Case 2 at the end of the proof of Lemma 7.6, $\|\omega\|=\|\mu\|$ and

$$
\left\|\bar{\Psi}_{a_{n}} \mu-\omega\right\| \rightarrow 0 \text { for some } F \text {-sequence. }
$$


Then $\omega=f \mu$ for some $f \in L(\mu)$ with $|f|=1$ a.e. $|\mu|$. We will identify $B$ with the collection of such $f$.

Now another subsequence has

$$
\bar{\Psi}_{a_{n}} \rightarrow f \text { a.e. }|\mu| \text {. }
$$

Let $S_{f}$ be where (4) holds and $|f|=1$. Then $S_{f}$ is a normal Borel subgroup, $|\mu|\left(S_{f}^{c}\right)=0$ and $f$ is a Borel homomorphism of $S_{f}$ into the unit circle.

Now $B$ is finite. Otherwise we would have $\omega_{i} \in B$ with $\omega_{i} \rightarrow \omega$ weakly for some $\omega \neq 0$. Using (3) and a diagonal process on the sequences converging to $\omega_{i}$ we could then find an $F$-sequence $\left\{a_{i}\right\}$ with

$$
\left\|\bar{\Psi}_{a_{i} \mu-\omega_{i}}\right\|<1 / 2 \text { and }\left\|\bar{\Psi}_{\alpha_{i} \mu-\omega}\right\|<1 / 2 \text {. }
$$

Since $\omega_{i} \in F(G)$ this would give $\omega_{i}=\omega$.

Let $p=\operatorname{card} B, M=\|\mu\|$ and $\delta=\delta(p, M)$ be the constant in Lemma 6.3. By using (3) there is an integer $N$ such that if $a=a_{1} a_{2} \cdots \in E(\mu)$ and $d\left(a_{i}\right)=$ 1 for $i \leq N$ then

$$
\left\|\left(\bar{\Psi}_{a}-f\right) \mu\right\|<\delta \text { for some } f \in B \text {. }
$$

Now let $T=\bigcap s$, over $f$ with $f \in B$. Each $f$ is a homomorphism on $T$ and $|\mu|^{\prime}\left(T^{c}\right)=0$. Let $K=\Pi_{1}^{N} G_{i}^{\prime}$ and $\lambda=\mu * \prod_{K^{\circ}}$. Then $\lambda \neq 0,\|\lambda\| \leq M$ and, since $K \subset T,|\lambda|\left(T^{c}\right)=0$. It also follows from $(6)$ that $\alpha \in E(\lambda)$ if and only if

$$
\left\|\left(\bar{\Psi}_{\alpha}-f\right) \lambda\right\|<\delta \text { for some } f \mu \in B \text {. }
$$

We can now apply Lemma 6.3 to $\lambda$ to obtain

$$
f_{1} \lambda * f_{2} \lambda * \cdots * f_{q} \lambda=A \pi_{H}
$$

where the $f_{i} \lambda$ are the distinct elements of $\{\lambda: f \in B\}, A \neq 0$ and $H$ is a closed normal subgroup.

Now $\Pi_{H} \mu \neq 0$ so that $H \cap L$ is open in $L$ by Theorem 3.2. On the other hand if $P$ is a normal Borel subgroup, $K \subset P$ and $\Pi_{P} \mu \neq 0$ then $\mu=\Pi_{p} \mu$ and so $\lambda=$ $\Pi_{P} \lambda$. Hence $\Pi_{P} \pi_{H}=\pi_{H}$ so that $P \cap H$ is open in $H$. This then implies $P$ $\cap L$ is open in $L$ so that $\mu$ is canonical by Lemma 7.6.

9. Connected groups. We can now use Theorem 2.2 to characterize $F(G)$ for connected $G$.

Lemma 9.1. If $G$ is a compact connected simple Lie group then $G$ satisfies conditions I and II.

Proof. Ragozin [4, Theorem 2.2] has shown that if $n=\operatorname{dimension} G$ and $\mu \in M^{Z}(G)$ is continuous then $\mu^{n} \in L_{1}(G)$. For $g \xi Z(G)$ let $\mu$ be given implicitly by 


$$
\int f d \mu=\int f\left(x g x^{-1}\right) d M_{G}(x) .
$$

It is easily seen that $\mu$ is a central continuous measure and $\hat{\mu}(a)=\bar{\Psi}_{a}(g)$ so that $\left(\mu^{n}\right)^{\wedge}(a)=\left(\bar{\Psi}_{a}(g)\right)^{n}$. By Ragozin's result and the Riemann-Lebesgue lemma it follows that

$$
\Psi_{a}(g) \rightarrow 0 \text { as } \alpha \rightarrow \infty(g \notin Z) \text {. }
$$

Condition I follows immediately. Also if $\{a\}$ is a sequence with $d(\alpha)=t$ then (2) and the dominated convergence theorem show

$$
1=\int\left|\chi_{a}\right|^{2} d \pi_{G}=t^{2} \pi_{G}(Z)+o(1) \text { as } a \rightarrow \infty \text {. }
$$

But then $\pi_{G}(Z) \neq 0$ so that $Z$ is open which is not possible since $G$ is connected Thus there are only finitely many $a$ with $d(a)=t$ which implies condition II.

Theorem 9.2. If $G$ is a connected compact group then every measure in $F(G)$ is canonical.

Proof. It is known (cf. [8, Chapitre V]) that a connected compact group $G$ is a factor group of a group $G^{*}=\Pi G_{i} \times A$ where $A$ is abelian and the $G_{i}$ are connected simple Lie groups. By Lemma 9.1 and Theorem 2.2 every measure in $F\left(G^{*}\right)$ is canonical. Since a measure in $F(G)$ can be considered as a measure in $F\left(G^{*}\right)$ the theorem follows.

10. An example. Unfortunately the characterization of $F(G)$ does not hold for all $G$ as the following simple example shows. Let $T \times T$ be the two dimensional torus and let $G$ be the semidirect product of $T \times T$ and $Z_{2}$ where $\delta\left(t_{1}, t_{2}\right)=\left(t_{2}, t_{1}\right) \delta$ for $\delta \in Z_{2}, \delta \neq e$. Let $\mu_{1}$ (resp. $\left.\mu_{2}\right)$ be the Haar measure of $T \times e$ (resp. $e \times T$ ). Then $\mu=\mu_{1}+\mu_{2} \in F(G)$ but $\mu$ is not canonical. That $\mu$ is not canonical is seen by noting that $\mu$ is singular to $\pi_{H}$ for every closed normal subgroup $H$ of $G$.

In this example $\mu$ is a sum of (noncentral) idempotents each of which is canonical with respect to its support group. It seems reasonable to conjecture that for any $G$ this is always the case.

Also if $\mu$ is canonical or as in the example then the conclusion of Lemma 4.2 holds for any Borel subgroup $H$ (whether or not it is closed and normal). It would be helpful to know whether this is true for any $\mu \in F(G)$.

It also seems likely that there is $\delta>0$ so that if $\mu \in F(G)$ and $\|\mu\|>1$ then $\|\mu\|>1+\delta$ (cf. Theorem 5.3). Otherwise some strange elements of $F$ could be obtained by taking infinite products of measures on product groups. 


\section{REFERENCES}

1. I. Amemiya and T. Itô, $A$ simple proof of the theorem of P. J. Cohen, Bull. Amer. Math. Soc. 70 (1964), 774-776. MR 29 \#4862.

2. P. J. Cohen, On a conjecture of Littlewood and idempotent measures, Amer. I. Math. 82 (1960), 191-212. MR 24 \#A3231.

3. F. P. Greenleaf, Norm decreasing homomorphisms of group algebras, Pacific J. Math. 15 (1965), 1187-1219. MR $33 \# 3117$.

4. D. L. Ragozin, Central measures on compact simple Lie groups, J. Functional Analysis (to appear).

5. D. Rider, Central idempotent measures on unitary groups, Canad. J. Math. 22 (1970), 719-725. MR 41 \#8929.

6. - Central idempotent measures on SIN groups, Duke Math. J. 38 (1971), 187-191. MR 42 \#6518.

7. W. Rudin, Fourier analysis on groups, Interscience Tracts in Pure and Appl. Math. no. 12, Interscience, New York, 1962. MR 27 \#2808.

8. A. Weil, L'integration dans les groupes topologiques et ses applications, Hermann, Paris, 1938.

DEPARTMENT OF MATHEMATICS, UNIVERSITY OF WISCONSIN, MADISON, WISCONSIN 53706 